\title{
Exact Solutions of Generalized Boussinesq-Burgers Equations and (2+1)-Dimensional Davey-Stewartson Equations
}

\author{
Isaiah Elvis Mhlanga and Chaudry Masood Khalique
}

International Institute for Symmetry Analysis and Mathematical Modelling,

Department of Mathematical Sciences, North-West University, Mafikeng Campus, Private Bag X 2046, Mmabatho 2735, South Africa

Correspondence should be addressed to Isaiah Elvis Mhlanga, isaiah.mhlanga@nwu.ac.za

Received 19 September 2012; Accepted 8 November 2012

Academic Editor: Asghar Qadir

Copyright (c) 2012 I. E. Mhlanga and C. M. Khalique. This is an open access article distributed under the Creative Commons Attribution License, which permits unrestricted use, distribution, and reproduction in any medium, provided the original work is properly cited.

We study two coupled systems of nonlinear partial differential equations, namely, generalized Boussinesq-Burgers equations and (2+1)-dimensional Davey-Stewartson equations. The Lie symmetry method is utilized to obtain exact solutions of the generalized Boussinesq-Burgers equations. The travelling wave hypothesis approach is used to find exact solutions of the $(2+1)$ dimensional Davey-Stewartson equations.

\section{Introduction}

Most nonlinear physical phenomena that appear in many areas of scientific fields such as plasma physics, solid state physics, fluid dynamics, optical fibers, mathematical biology, and chemical kinetics can be modelled by nonlinear partial differential equations (NLPDEs). The investigation of exact travelling wave solutions of these NLPDEs is important for the understanding of most nonlinear physical phenomena and possible applications. To address this issue, various methods for finding travelling wave solutions to NLPDEs have been proposed. Some of the most important methods include homogeneous balance method [1], the ansatz method $[2,3]$, variable separation approach [4], inverse scattering transform method [5], Bäcklund transformation [6], Darboux transformation [7], Hirota's bilinear method [8], the $\left(G^{\prime} / G\right)$-expansion method [9], the reduction mKdV equation method [10], the tri-function method [11, 12], the projective Riccati equation method [13], the sine-cosine method [14, 15], the Jacobi elliptic function expansion method [16, 17], the F-expansion method [18], the exp-function expansion method [19], and Lie symmetry method [20-24]. 
In this paper, we study two systems of nonlinear partial differential equations, namely, generalized Boussinesq-Burgers equations and (2+1)-dimensional Davey-Stewartson equations. We employ the Lie symmetry method and the travelling wave variable approach to obtain exact solutions to both these systems.

The Lie symmetry approach is one of the most effective methods to determine solutions of nonlinear partial differential equations. Sophus Lie (1842-1899), with the inspiration from Galois' theory for solving algebraic equations, discovered this method which is known today as Lie group analysis. He showed that many of the known methods of integration of ordinary differential equations could be derived in a systematic manner using his theory of continuous transformation groups. In the past few decades, a considerable amount of development has been made in symmetry methods for differential equations. This is evident by the number of research papers, books, and many new symbolic softwares devoted to the subject [20-24]. The travelling wave variable approach converts the NLPDEs into a nonlinear ordinary differential equations and is often useful in obtaining exact solutions of the partial differential equations.

\section{The Generalized Boussinesq-Burgers Equations}

We first consider the generalized Boussinesq-Burgers equations [25] given by

$$
\begin{gathered}
u_{t}+a u u_{x}+b v_{x}=0, \\
v_{t}+c(u v)_{x}+d u_{x x x}=0,
\end{gathered}
$$

where $a, b, c$, and $d$ are real nonzero constants. These equations arise in the study of fluid flow and describe the propagation of shallow water waves, where $x$ and $t$ represent the normalized space and time, respectively. Here $u(x, t)$ represents the horizontal velocity and at the leading order it is the depth averaged horizontal field, while $v(x, t)$ denotes the height of the water surface above the horizontal level at the bottom [25].

The Boussinesq-Burgers equations given by (2.1)-(2.2) will be solved by the Lie symmetry approach. The symmetry group of the generalized Boussinesq-Burgers equations (2.1)-(2.2) will be generated by the vector field given by

$$
X=\xi^{1}(t, x, u, v) \frac{\partial}{\partial t}+\xi^{2}(t, x, u, v) \frac{\partial}{\partial x}+\eta^{1}(t, x, u, v) \frac{\partial}{\partial u}+\eta^{2}(t, x, u, v) \frac{\partial}{\partial v} .
$$

Applying the third prolongation $\operatorname{pr}^{(3)} X[21]$ to (2.1)-(2.2) and solving the resultant overdetermined system of linear partial differential equations, one obtains the following three Lie point symmetries:

$$
\begin{gathered}
X_{1}=\frac{\partial}{\partial t^{\prime}} \\
X_{2}=\frac{\partial}{\partial x} \\
X_{3}=-2 t \frac{\partial}{\partial t}-x \frac{\partial}{\partial x}+u \frac{\partial}{\partial u}+2 v \frac{\partial}{\partial v} .
\end{gathered}
$$


We now consider the symmetry $X_{1}+v X_{2}$, where $v$ is an arbitrary constant. This symmetry gives rise to the group-invariant solution as

$$
u=F(z), \quad v=G(z),
$$

where $z=x-v t$ is an invariant of the symmetry $X_{1}+v X_{2}$. Substitution of (2.5) into (2.1)-(2.2) results in the system of ordinary differential equations as

$$
\begin{gathered}
a F(z) F^{\prime}(z)-v F^{\prime}(z)+b G^{\prime}(z)=0, \\
d F^{\prime \prime \prime}(z)+c G(z) F^{\prime}(z)+c F(z) G^{\prime}(z)-v G^{\prime}(z)=0 .
\end{gathered}
$$

Integration of (2.6) with respect to $z$ yields

$$
\frac{1}{2} a F(z)^{2}-v F(z)+b G(z)=0
$$

where the constant of integration is chosen to be zero, since we are looking for a soliton solution. Solving for $G(z)$, we obtain

$$
G(z)=\frac{2 v F(z)-a F(z)^{2}}{2 b}
$$

Substituting this value of $G(z)$ into (2.7) gives the third-order nonlinear ordinary differential equation as

$$
2 b d F^{\prime \prime \prime}(z)-3 a c F(z)^{2} F^{\prime}(z)+2 v(a+2 c) F(z) F^{\prime}(z)-2 v^{2} F^{\prime}(z)=0,
$$

which can be integrated twice to obtain

$$
F^{\prime}(z)^{2}-\frac{a c}{4 b d} F(z)^{4}+\frac{v(a+2 c)}{3 b d} F(z)^{3}-\frac{v^{2}}{b d} F(z)^{2}=0
$$

Here again the constants of integration are taken to be zero for the same reason as given above. Integrating (2.11) and reverting back to our original variables, we obtain

$$
\begin{gathered}
u(x, t)=\frac{12 v}{2(a+2 c)+\alpha \cosh (\gamma z+\delta)-\beta \sinh (\gamma z+\delta)} \\
v(x, t)=\frac{12 v^{2}[-6 a+2(a+2 c)+\alpha \cosh (\gamma z+\delta)-\beta \sinh (\gamma z+\delta)]}{b[2(a+2 c)+\alpha \cosh (\gamma z+\delta)-\beta \sinh (\gamma z+\delta)]^{2}}
\end{gathered}
$$




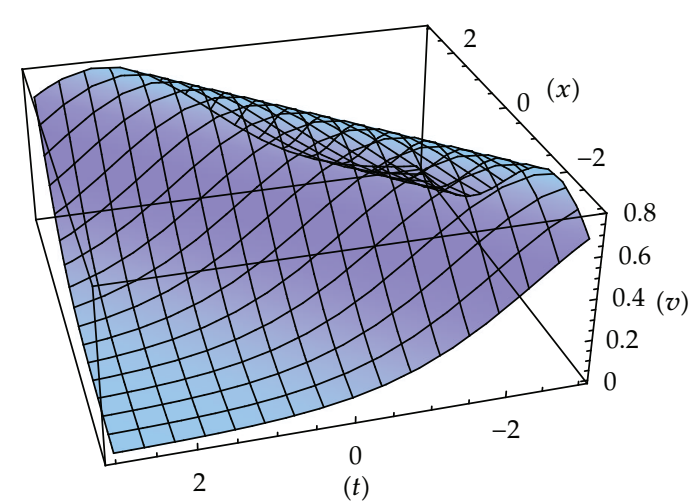

(a)

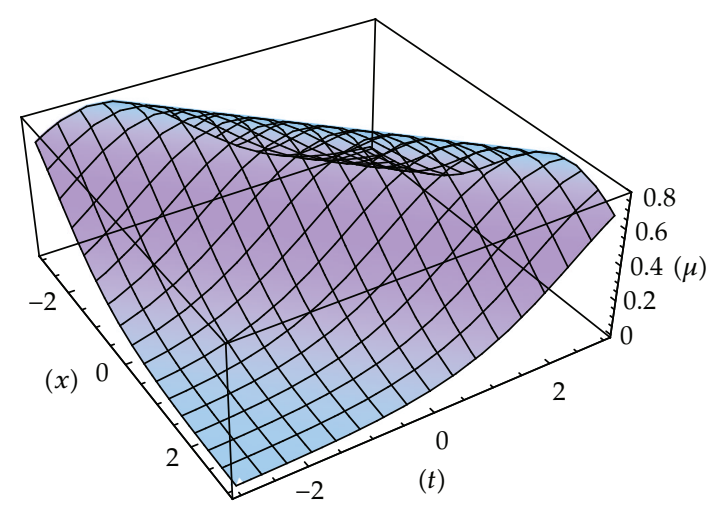

(b)

Figure 1: Profile of the solitary wave solution (2.12)-(2.13).

where $\alpha, \beta, \gamma$, and $\delta$ are constants given by

$$
\begin{gathered}
\alpha=a^{2}-5 a c+4 c^{2}+1, \\
\beta=a^{2}-5 a c+4 c^{2}-1, \\
\gamma=\frac{v}{\sqrt{b d}}, \\
\delta=2 \sqrt{3} c v, \\
z=x-v t .
\end{gathered}
$$

A profile of the solution (2.12)-(2.13) is given in Figure 1.

\section{The (2+1)-Dimensional Davey-Stewartson Equations}

The (2+1)-dimensional Davey-Stewartson equations

$$
\begin{gathered}
i u_{t}+u_{x x}-u_{y y}-2|u|^{2} u-2 u v=0, \\
v_{x x}+v_{y y}+2\left(|u|^{2}\right)_{x x}=0
\end{gathered}
$$

were first introduced by Davey and Stewartson in 1974 [26]. This system of equations is completely integrable and is often used to describe the long-time evolution of a twodimensional wave packet [27-29].

We first transform the (2+1)-dimensional Davey-Stewartson equations (3.1)-(3.2) to a system of nonlinear ordinary differential equations in order to derive its exact solutions.

We make the following transformation:

$$
u=e^{i \theta} u(\xi), \quad v=v(\xi), \quad \theta=p x+q y+r t, \quad \xi=k x+c y+d t,
$$


where $p, q, r, k, c$, and $d$ are real constants. Using this transformation, the (2+1)-dimensional Davey-Stewartson equations (3.1)-(3.2) transform to

$$
\begin{gathered}
\left(-r-p^{2}+q^{2}\right) u+i(d+2 p k-2 c q) u^{\prime}+\left(k^{2}-c^{2}\right) u^{\prime \prime}-2 u^{3}-2 u v=0 \\
\left(k^{2}+c^{2}\right) v^{\prime \prime}+2\left(u^{2}\right)^{\prime \prime}=0 .
\end{gathered}
$$

Integration of (3.5) twice and taking the constants of integration to be zero, one obtains

$$
v=\frac{-2 u^{2}}{k^{2}+c^{2}}
$$

Now substituting (3.6) into (3.4), we get

$$
u^{\prime \prime}=\left(\frac{r+p^{2}-q^{2}}{k^{2}-c^{2}}\right) u+\left(\frac{2 k^{2}+2 c^{2}-4}{k^{4}-c^{4}}\right) u^{3}
$$

which can be written in the following form:

$$
u^{\prime \prime}=A u+B u^{3},
$$

where

$$
A=\frac{r+p^{2}-q^{2}}{k^{2}-c^{2}}, \quad B=\frac{2 k^{2}+2 c^{2}-4}{k^{4}-c^{4}} .
$$

Solving (3.8), with the aid of Mathematica, we obtain the following solution:

$$
u= \pm \frac{1}{P_{2}} i \operatorname{sn}\left(P_{1} \mid \omega\right)
$$

where $\operatorname{sn}\left(P_{1} \mid \omega\right)$ is a Jacobian elliptic function of the sine-amplitude [30], and

$$
\begin{gathered}
P_{1}=\frac{\sqrt{\left(\sqrt{A^{2}-2 B c_{1}}-A\right)\left(z+c_{2}\right)^{2}}, \quad P_{2}=\sqrt{\frac{B}{\sqrt{A^{2}-2 B c_{1}}+A}},}{\sqrt{2}}, \\
\omega=\frac{-B c_{1}+A\left(\sqrt{A^{2}-2 B c_{1}}+A\right)}{B c_{1}}
\end{gathered}
$$




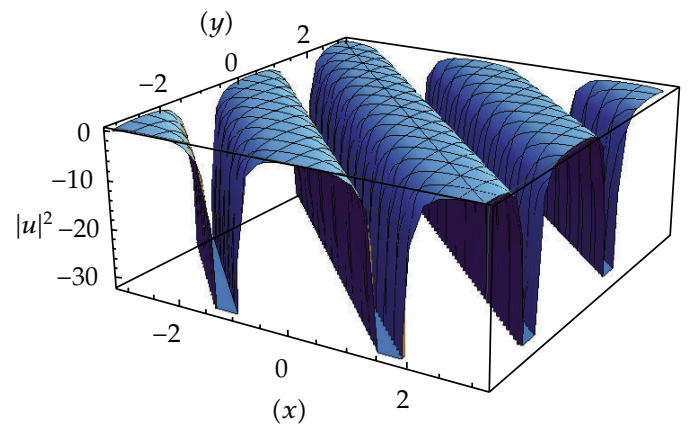

(a)

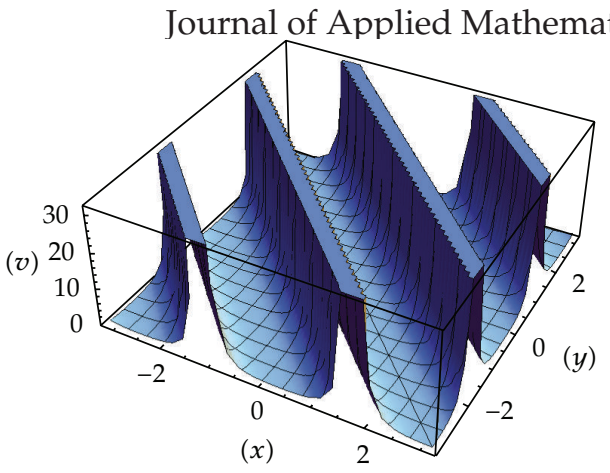

(b)

Figure 2: Profile of the solution (3.12).

is the modulus of the elliptic function with $0<\omega<1$. Here $c_{1}$ and $c_{2}$ are constants of integration. Reverting back to our original variables, we can now write the solution of our $(2+1)$ dimensional Davey-Stewartson equations as

$$
u(x, y, t)= \pm \frac{1}{P_{2}} i \operatorname{sn}\left(P_{1} \mid \omega\right)
$$

where

$$
P_{1}=\frac{\sqrt{\left(\sqrt{A^{2}-2 B c_{1}}-A\right)\left(k x+c y+d t+c_{2}\right)^{2}}}{\sqrt{2}},
$$

$\omega$, and $P_{2}$ are as above.

Now $v(x, y, t)$ can be obtained from (3.6).

It should be noted that the solution (3.12) is valid for $0<\omega<1$ and as $\omega$ approaches zero, the solution becomes the normal sine function, $\sin z$, and as $\omega$ approaches 1 , the solution tends to the tanh function, $\tanh z$.

The profile of the solution (3.12) is given in Figure 2.

\section{Conclusion}

In this paper, we studied two systems of nonlinear partial differential equations. Firstly, we obtained exact solutions of the generalized Boussinesq-Burgers equations given by (2.1)-(2.2) using the Lie symmetry method. The solutions obtained were travelling wave solutions. Secondly, we found exact solutions of the $(2+1)$-dimensional Davey-Stewartson equations (3.1)-(3.2) using the travelling wave hypothesis. The Davey-Stewartson system was first transformed to a system of nonlinear ordinary differential equations, which was then solved to obtain the exact solutions.

\section{Acknowledgments}

I. E. Mhlanga and C. M. Khalique would like to thank the Organizing Committee of the Symmetries, Differential Equations, and Applications: Galois Bicentenary Conference (SDEA2012) for their kind hospitality during the conference. 


\section{References}

[1] M. Wang, Y. Zhou, and Z. Li, "Application of a homogeneous balance method to exact solutions of nonlinear equations in mathematical physics," Physics Letters A, vol. 216, no. 1-5, pp. 67-75, 1996.

[2] J. Hu, "Explicit solutions to three nonlinear physical models," Physics Letters A, vol. 287, no. 1-2, pp. 81-89, 2001.

[3] J. Hu and H. Zhang, "A new method for finding exact traveling wave solutions to nonlinear partial differential equations," Physics Letters A, vol. 286, no. 2-3, pp. 175-179, 2001.

[4] S.-Y. Lou and J. Lu, "Special solutions from the variable separation approach: the Davey-Stewartson equation," Journal of Physics A, vol. 29, no. 14, pp. 4209-4215, 1996.

[5] M. J. Ablowitz and P. A. Clarkson, Solitons, Nonlinear Evolution Equations and Inverse Scattering, vol. 149 of London Mathematical Society Lecture Note Series, Cambridge University Press, Cambridge, UK, 1991.

[6] C. H. Gu, Soliton Theory and Its Application, Zhejiang Science and Technology Press, Zhejiang, China, 1990.

[7] V. B. Matveev and M. A. Salle, Darboux Transformations and Solitons, Springer Series in Nonlinear Dynamics, Springer, Berlin, Germany, 1991.

[8] R. Hirota, The Direct Method in Soliton Theory, vol. 155 of Cambridge Tracts in Mathematics, Cambridge University Press, Cambridge, UK, 2004.

[9] E. M. E. Zayed and K. A. Gepreel, “The $\left(G^{\prime} / G\right)$-expansion method for finding traveling wave solutions of nonlinear partial differential equations in mathematical physics," Journal of Mathematical Physics, vol. 50, no. 1, Article ID 013502, 2009.

[10] Z. Yan, "A reduction mKdV method with symbolic computation to construct new doubly-periodic solutions for nonlinear wave equations," International Journal of Modern Physics C, vol. 14, no. 5, pp. 661-672, 2003.

[11] Z. Yan, "The new tri-function method to multiple exact solutions of nonlinear wave equations," Physica Scripta, vol. 78, no. 3, Article ID 035001, 2008.

[12] Z. Yan, "Periodic, solitary and rational wave solutions of the 3D extended quantum Zakharov-Kuznetsov equation in dense quantum plasmas," Physics Letters A, vol. 373, no. 29, pp. 2432-2437, 2009.

[13] D. Lu and B. Hong, "New exact solutions for the (2+1)-dimensional generalized Broer-Kaup system," Applied Mathematics and Computation, vol. 199, no. 2, pp. 572-580, 2008.

[14] A.-M. Wazwaz, "The tanh and the sine-cosine methods for compact and noncompact solutions of the nonlinear Klein-Gordon equation," Applied Mathematics and Computation, vol. 167, no. 2, pp. 11791195, 2005.

[15] Z. Yan and H. Zhang, "New explicit solitary wave solutions and periodic wave solutions for Whitham-Broer-Kaup equation in shallow water," Physics Letters A, vol. 285, no. 5-6, pp. 355-362, 2001.

[16] D. Lü, “Jacobi elliptic function solutions for two variant Boussinesq equations," Chaos, Solitons and Fractals, vol. 24, no. 5, pp. 1373-1385, 2005.

[17] Z. Yan, "Abundant families of Jacobi elliptic function solutions of the $(2+1)$-dimensional integrable Davey-Stewartson-type equation via a new method," Chaos, Solitons and Fractals, vol. 18, no. 2, pp. 299-309, 2003.

[18] M. Wang and X. Li, “Extended F-expansion method and periodic wave solutions for the generalized Zakharov equations," Physics Letters A, vol. 343, no. 1-3, pp. 48-54, 2005.

[19] J.-H. He and X.-H. Wu, "Exp-function method for nonlinear wave equations," Chaos, Solitons $\mathcal{E}$ Fractals, vol. 30, no. 3, pp. 700-708, 2006.

[20] G. W. Bluman and S. Kumei, Symmetries and Differential Equations, vol. 81 of Applied Mathematical Sciences, Springer, New York, NY, USA, 1989.

[21] P. J. Olver, Applications of Lie Groups to Differential Equations, vol. 107 of Graduate Texts in Mathematics, Springer, New York, NY, USA, 2nd edition, 1993.

[22] N. H. Ibragimov, CRC Handbook of Lie Group Analysis of Differential Equations, vol. 1-3, CRC Press, Boca Raton, Fla, USA, 1994.

[23] L. V. Ovsiannikov, Group Analysis of Differential Equations, Academic Press, New York, NY, USA, 1982.

[24] A. R. Adem and C. M. Khalique, "Symmetry reductions, exact solutions and coservation laws of a new coupled KdV system," Communications in Nonlinear Science and Numerical Simulation, vol. 17, pp. 34653475, 2012. 
[25] P. Wang, B. Tian, W.-J. Liu, X. Lü, and Y. Jiang, "Lax pair, Bäcklund transformation and multi-soliton solutions for the Boussinesq-Burgers equations from shallow water waves," Applied Mathematics and Computation, vol. 218, no. 5, pp. 1726-1734, 2011.

[26] A. Davey and K. Stewartson, "On three-dimensional packets of surface waves," Proceedings of the Royal Society A, vol. 338, pp. 101-110, 1974.

[27] S. A. El-Wakil, M. A. Abdou, and A. Elhanbaly, "New solitons and periodic wave solutions for nonlinear evolution equations," Physics Letters A, vol. 353, no. 1, pp. 40-47, 2006.

[28] E. Fan and J. Zhang, "Applications of the Jacobi elliptic function method to special-type nonlinear equations," Physics Letters A, vol. 305, no. 6, pp. 383-392, 2002.

[29] A. Bekir and A. C. Cevikel, "New solitons and periodic solutions for nonlinear physical models in mathematical physics," Nonlinear Analysis: Real World Applications, vol. 11, no. 4, pp. 3275-3285, 2010.

[30] I. S. Gradshteyn and I. M. Ryzhik, Table of Integrals, Series, and Products, Academic Press, New York, NY, USA, 7th edition, 2007. 


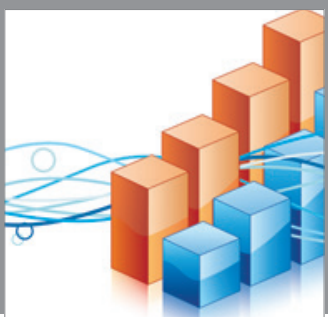

Advances in

Operations Research

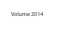

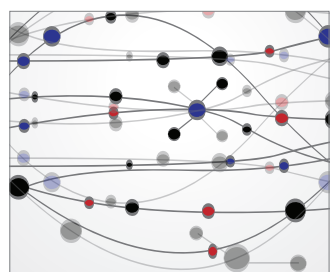

\section{The Scientific} World Journal
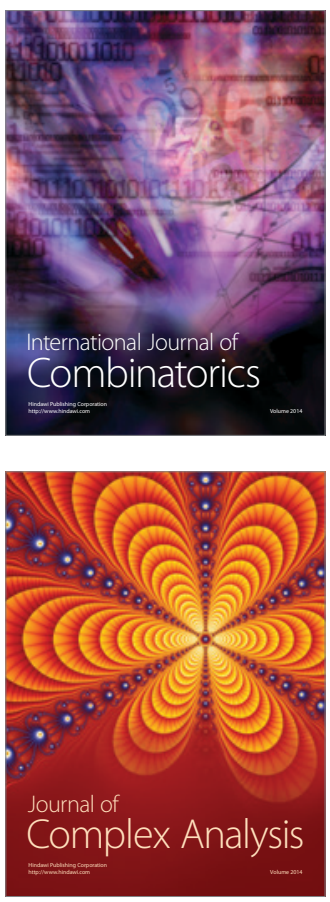

International Journal of

Mathematics and

Mathematical

Sciences
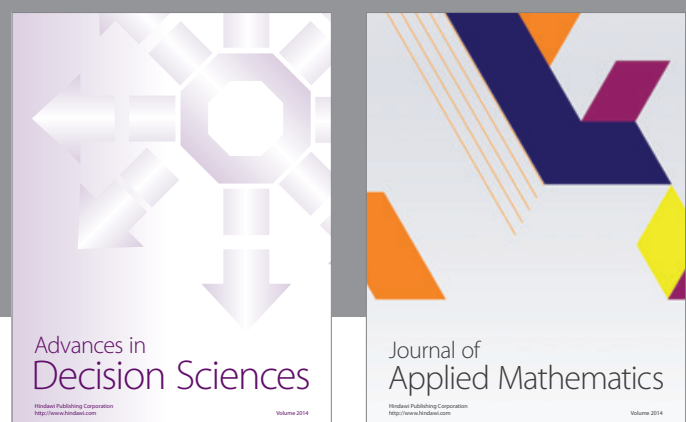

Journal of

Applied Mathematics
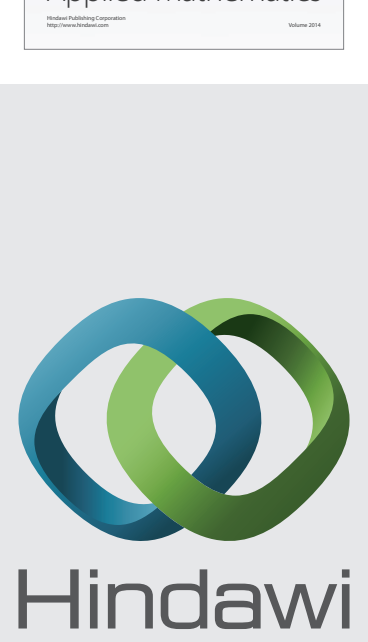

Submit your manuscripts at http://www.hindawi.com
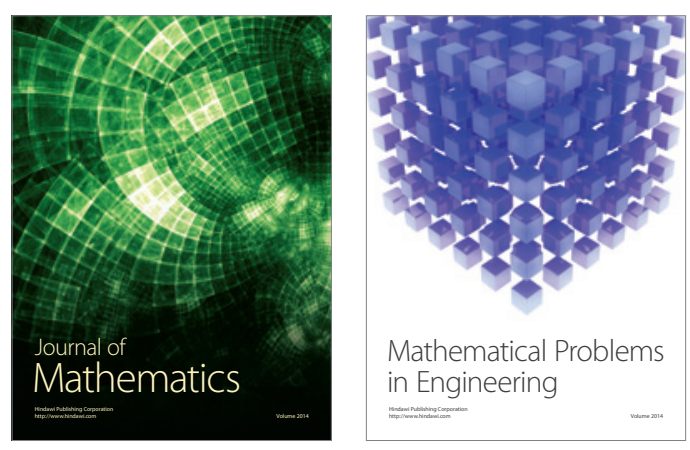

Mathematical Problems in Engineering
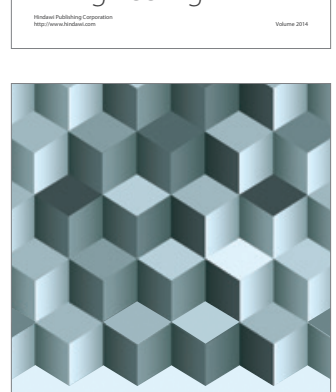

Journal of

Function Spaces
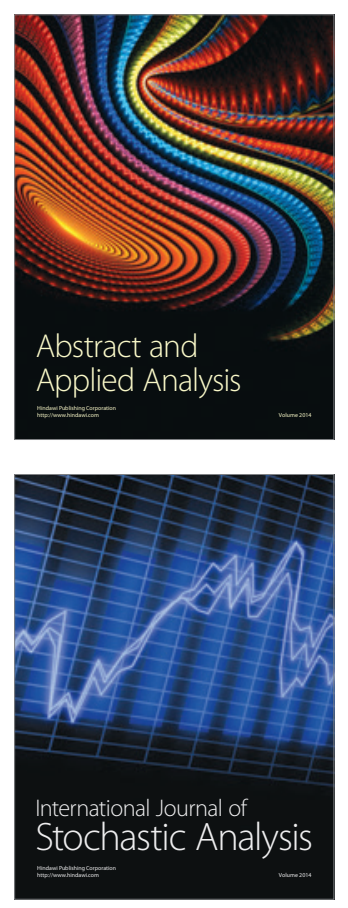

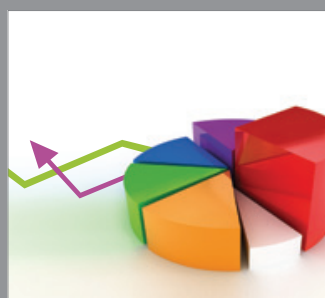

ournal of

Probability and Statistics

Promensencen
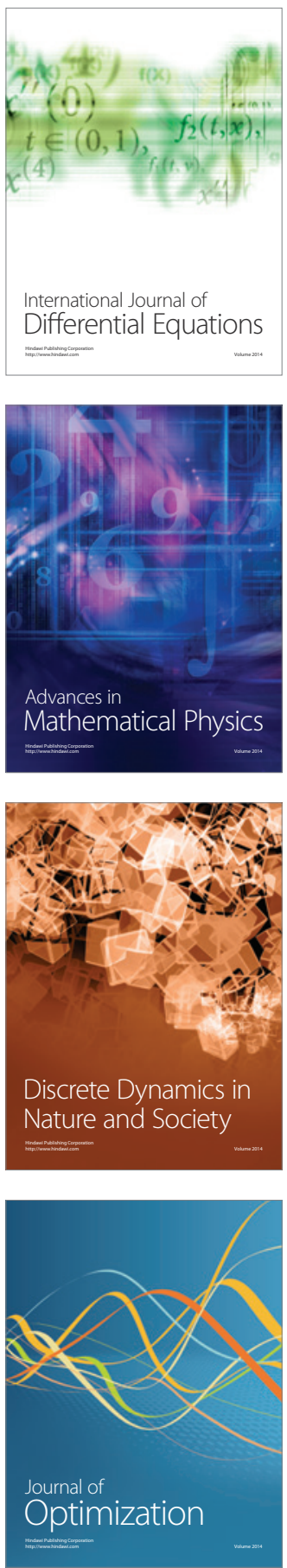